\title{
SURFACE AND BULK HARMONIC GENERATION IN THE OPAQUE REGION OF GaAs
}

\section{Laura Rodríguez Suñé}

Supervised by Dr./Prof. Crina Cojocaru (UPC) and Jose Trull (UPC) 


\title{
Surface and bulk harmonic generation in the opaque region of GaAs
}

\author{
Laura Rodríguez Suñé \\ Nonlinear Dynamics, Nonlinear Optics and Lasers Research Group (DONLL), Univ. \\ Politcnica de Catalunya, Rambla Sant Nebridi 22, 08222 Terrassa (Barcelona, Spain)
}

\begin{abstract}
.
We demonstrate second and third harmonic generation from a GaAs substrate, far from the phase matching and well-below the absorption edge, in both transmission and reflection geometries. The pump is tuned in the transparency range, while the SH and the TH signals are tuned in the opaque spectral range of GaAs. We find that the polarization of the generated signals is sensitive to the polarization of the pump. The experimental results are contrasted with numerical simulations that account for both surface and bulk contributions and show that the bulk-generated SH components are less intense than surface-generated SH signals.
\end{abstract}

Keywords: Nonlinear optics, Harmonic generation, Phase lock

\section{Introduction}

Nonlinear (NL) optics is the study of phenomena that occur as a consequence of the modification of the optical properties of a material due to the presence of intense light. Its traditional topics cover different types of parametric processes, such as second harmonic generation (SHG).

In NL phenomena, the polarization of the material system depends on the strength of the applied optical field following the well-known equation:

$$
\begin{aligned}
P(t) & =\epsilon_{0}\left[\chi^{(1)} E(t)+\chi^{(2)} E^{2}(t)+\chi^{(3)} E^{3}(t)+\ldots\right] \\
& \equiv P^{(1)}(t)+P^{(2)}(t)+P^{(3)}(t)+\ldots
\end{aligned}
$$

where $\chi^{(1)}$ is the linear susceptibility, $\chi^{(2)}$ and $\chi^{(3)}$ are known as the second- and thirdorder NL susceptibilities, and $\epsilon_{0}$ is the permittivity of free space. Interactions due to $P^{(2)}(t)=\epsilon_{0} \chi^{(2)} E^{2}(t)$ can occur only in non centro-symmetric crystals. In SHG two photons at frequency $\omega$ interact with a NL material and generate a new photon at $2 \omega$. SHG can occur in the bulk of non centro-symetric materials as well as at the surface of all materials, due to breaking of translational symmetry. On the other hand, third-order NL interactions can occur in both centro-symmetric and non centro-symmetric crystals. In Eq.(1) we have taken the fields $E$ and $P$ to be scalar quantities. However, one generally need to account for the vector nature of the fields. Then, the susceptibility becomes a tensor that couples the different components of the field and polarization. Under the Kleinman's symmetry condition, assuming a lossless NL medium, and 
considering the symmetry of (001) GaAs, the polarization of the SH generated field $(2 \omega)$ as a function of the fundamental field $(\mathrm{FF}), \omega$, may be written as follows:

$$
\left(\begin{array}{c}
P_{x}^{(2)}(2 \omega) \\
P_{y}^{(2)}(2 \omega) \\
P_{z}^{(2)}(2 \omega)
\end{array}\right)=2\left(\begin{array}{cccccc}
0 & 0 & 0 & d_{14} & 0 & 0 \\
0 & 0 & 0 & 0 & d_{14} & 0 \\
0 & 0 & 0 & 0 & 0 & d_{14}
\end{array}\right)\left(\begin{array}{c}
E_{x}^{2}(\omega) \\
E_{y}^{2}(\omega) \\
E_{z}^{2}(\omega) \\
2 E_{y}(\omega) E_{z}(\omega) \\
2 E_{x}(\omega) E_{z}(\omega) \\
2 E_{x}(\omega) E_{y}(\omega)
\end{array}\right)
$$

where $d_{14}$ is the only non-banishing element of a matrix $d$ referred to as the $N L$ coefficient tensor which is related to the susceptibility tensor: $d=\frac{1}{2} \epsilon_{0} \chi^{(2)}(d \mathrm{in} \mathrm{m} / \mathrm{V})$.

Eq.(2) is a general expression that allows an electric field to interact with a NL medium and to generate a SH field. The SHG efficiency is given by Eq. (3), where $n_{1}$ is the index of refraction of the linear medium, $n_{2}$ is the index of refraction of the NL material, $d_{\text {eff }}$ is called the d effective value, $I_{\omega}(0)$ is the intensity of the FF at the entrance of the material, $L$ is the thickness of the sample, and $\Delta k$ is called the phase mismatch factor: $\Delta \vec{k}=\vec{k}_{2}-2 \vec{k}_{1}$, where $\vec{k}_{1}$ and $\vec{k}_{2}$ are the wave-vectors of the fundamental and the SH fields, respectively.

$$
e_{S H G}=\frac{1}{2} \frac{(2 \omega)^{2} d_{e f f}^{2}}{n_{1}^{2} n_{2} \epsilon_{0} c^{3}} I_{\omega}(0) L^{2} \operatorname{sinc}^{2}\left(\frac{\Delta k}{2} L\right)
$$

Perfect phase matching (PM) occurs when $\Delta \vec{k}=0$, a condition that requires both $\mathrm{FF}$ and $\mathrm{SH}$ fields to have the same phase velocities. Due to material dispersion, the FF and the SH fields generally experience different group velocities. The distance at which the $\mathrm{SH}$ field is no longer under the spatial influence of the FF pulse due to differences in group velocities is called the walk-off distance, and it is better observed when $\Delta \vec{k} \neq 0$. Under phase mismatched conditions, when a pump pulse crosses an interface between a linear and a NL medium there are always three generated $\mathrm{SH}$ components. One component is generated backward (reflection) into the linear medium; the other two components are generated forward. This was discussed first in [1] where a mathematical treatment provided a general solution of the SH field that showed two contributions, one traveling with the characteristic group velocity expected at the second harmonic frequency, and a second component that instead appeared to travel with the group velocity of the FF.

It is well-known that these forward-propagating components arise from the homogeneous $(\mathrm{HOM})$ and inhomogeneous (INHOM) solutions of the wave equation at the $\mathrm{SH}$ frequency. The HOM component travels with the group velocity given by material dispersion: $k(2 \omega)=k_{0}(2 \omega) n(2 \omega)$, where $k_{0}(2 \omega)=2 \omega / c$ is the wave-number expression for the $\mathrm{SH}$ in vaccum; while the INHOM component is captured by the pump pulse and experiences its same effective dispersion: $2 k_{0}(\omega) n(\omega)$, where $k_{0}(\omega)=\omega / c$ is the wave-number of the FF in vacuum. In this case it is said that the $\mathrm{SH}$ pulse is phase locked (PL). This phenomenon was firts observed in [2].

If absorption is present at the $\mathrm{SH}$ wavelength the HOM component is absorbed, while the PL solution can co-propagate with the pump and exit the medium. Harmonic generation in absorbing materials has previously been considered in the context of measuring nonlinear coefficients but it has not been as widely studied as in the transparency 
frequency range.

In [3] the inhibition of absorption for femtosecond, $\mathrm{SH}$ and $\mathrm{TH}$ generated signals in a bulk GaAs substrate was experimentally observed. Fig. 1 shows the real and imaginary parts of the permittivity of GaAs reported in [4]. One may surmise that the medium is transparent for wavelengths above $900 \mathrm{~nm}$, and strongly absorptive below it. This means that if the FF is pumped at $1064 \mathrm{~nm}$, the $\mathrm{SH}(532 \mathrm{~nm})$ and the TH $(355 \mathrm{~nm})$ signals are tuned well below the absorption edge of GaAs.

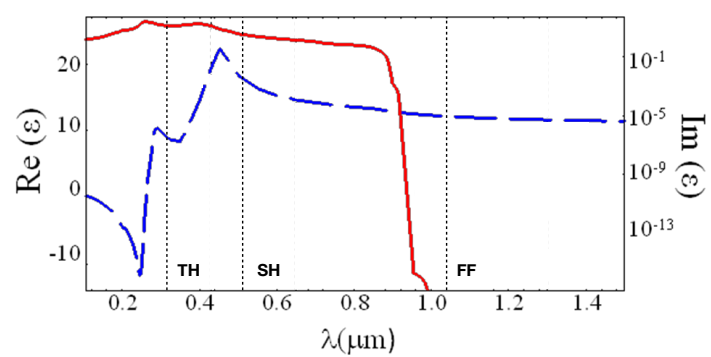

Figure 1: Real (blue) and imaginary (red) part of the permittivity of GaAs.

The SH fields can also be generated at the surface of the medium as a result of breaking of translational symmetry. This phenomena can be understood following the theoretical approach outlined in [5]. In general, nonlinear sources may be specified in terms of a complex dielectric function described by a combined Drude-Lorentz, which contains a mix of free and bound electrons having one or more resonances. For typical GaAs substrates, free carrier doping (n- or p-type) ranges from $10^{14} \mathrm{~cm}^{-3}-10^{17} \mathrm{~cm}^{-3}$, so that the Drude portion may be neglected. Therefore, in our case only bound electrons are assumed to play a role in surface SHG.

Neglecting for the moment NL restoring forces $\left(\chi^{(2)}, \chi^{(3)}\right.$, etc $)$, Newton's second law for one species of bound electrons leads to the following effective polarization equation for bound charges:

$$
\ddot{\overrightarrow{P_{b}}}+\gamma_{b} \dot{\vec{P}}_{b}+\omega_{0, b}^{2} \vec{P}_{b}=\frac{n_{0, b} e^{2}}{m_{b}^{*}} \vec{E}+\frac{e}{m_{b}^{*} c} \dot{\vec{P}}_{b} \times \vec{H}
$$

where $\vec{P}_{b}$ is the polarization, $\dot{\vec{P}}_{b}$ is the bound current density, $\vec{H}$ is the magnetic field, $e$ is the charge of the electron, $m_{b}^{*}$ is the bound electron's effective mass, and $c$ is the speed of light. When developing Eq. (4), the following expressions for the SH polarization envelope function $([5],[6])$ is found in the undepleted pump approximation:

$$
\ddot{\vec{P}}_{b, 2 \omega}+\tilde{\gamma}_{b} \dot{\vec{P}}_{b, 2 \omega}+\tilde{\omega}_{0, b}^{2} \vec{P}_{b, 2 \omega} \approx \frac{n_{0, b} e^{2} \lambda_{0}^{2}}{m_{b}^{*} c^{2}} \vec{E}_{2 \omega}+\frac{e \lambda_{0}}{m_{b}^{*} c^{2}}\left(\vec{E}_{\omega} \vec{\nabla} \vec{P}_{b, \omega}+\left(\dot{\vec{P}}_{b, \omega}-i \omega \vec{P}_{b, \omega}\right) \times \vec{H}_{\omega}\right)
$$

where $\lambda_{0}=1 \mu m$ and some coefficients have been scaled: $\gamma_{b, N \omega}=\lambda_{0}\left(\gamma_{b}-N i \omega\right) / c$, $\tilde{\omega}_{0, b, N \omega}^{2}=\lambda_{0}\left(\omega_{0}^{2}-(N \omega)^{2}+i \gamma_{b} N \omega\right) / c$ where $N=2$ is an integer that denotes the $\mathrm{SH}$.

Eq.(5) suggests that in the absence of bulk nonlinearities, SHG comes about from the gradient of the polarization, which is non-zero at the surface, and the magnetic field (Lorentz force), which has both surface and bulk components. Eq. (5) describes bound charges that give rise to SHG in metallic structures as well as in dielectrics and 
semiconductors of arbitrary geometry, and are valid in the ultrashort pulse regime. NL restoring forces may be introduced into Eq. (5) to generalize it and gain the ability to account for harmonic generation in dielectric and semiconductor structures alike. This may be achieved by introducing a NL polarization in the usual way, i.e. Eq. (1). The total second order NL polarization may be written in a simplified manner as follows:

$$
\vec{P}_{N L}(2 \omega) \approx \chi^{(2)}(\omega) \vec{E}^{2}+\chi^{(3)}(\omega) \vec{E}^{3}+\frac{e \lambda_{0}}{m_{b}^{*} c^{2}}\left(\vec{E}_{\omega} \vec{\nabla}_{P_{b, \omega}}+\left(\dot{\vec{P}}_{b, \omega}-i \omega \vec{P}_{b, \omega}\right) \times \vec{H}_{\omega}\right)
$$

where $\chi^{(2)}$ and $\chi^{(3)}$ are tensor quantities. The first and second terms on the right hand side of Eq. (6) include the contribution of the bulk to SHG and THG, respectively, the gradient term represents the surface contribution and the remaining terms are the Lorentz contribution.

In this thesis we will study the PL SH and TH signals generated when a $1064 \mathrm{~nm}$ pumped laser interacts with a GaAs substrate. The SH and $\mathrm{TH}$ signals are generated at $532 \mathrm{~nm}$ and $355 \mathrm{~nm}$ respectively, well-below the absorption edge of GaAs. A discussion about where this signals come from (bulk or surface SHG) will be included too as well as a comparison of the experimental results with theoretical simulations.

\section{Experimental setup}

As NL material we have chosen a GaAs substrate of $500 \mu m$ thick, grown in the 001 direction. Fig. $2 \mathrm{~b}$ shows the direction of the three crystallographic axes, as well as the angle of incidence of the FF beam, $\theta$. Following this reference frame, the electric field for the TE-polarized light can be written as $\vec{E}_{T E}=E_{T E 0} \vec{i}$ and for the TM-polarized light as $\vec{E}_{T M}=E_{T M 0}(\cos \theta \vec{j}+\sin \theta \vec{k})$. If the polarization of the incident light is a sum of TE and TM-polarized light, taking $\phi$ as the angle of rotation of the electric field, and taking into account Eq. (2), the polarization vector of the SH field can be calculated:

$$
\vec{P}(2 \omega)=d E_{0}^{2}\left(\cos ^{2} \phi \cos \theta \sin \theta \vec{i}+\sin \phi \cos \phi \sin \theta \vec{j}+\sin \phi \cos \phi \cos \theta \vec{k}\right)
$$

Eq. (7) tells us that, considering only the bulk contribution $\left(\chi^{(2)}\right)$ in the generation of the SH field and considering a particular angle of incidence, the TE and TM-polarized SH efficiency would be proportional to $\cos ^{4} \phi$ and $\sin ^{2}(2 \phi)$, respectively. Nonetheless, if the gradient term of Eq. (6) is developed it can be shown that the dependence is the opposite. In the next section we will analyze our measurements and we will see that they can be understood taking into account surface SHG (surface and Lorentz terms of Eq. (6)).

For a complete study of the harmonic signals generated by the GaAs substrate we have designed and build a setup capable of measuring the SH both in transmission and reflection. The schematic representation for each configuration is shown in Figs. 2a and $2 \mathrm{~b}$, respectively. We have used a Nd:YAG laser tuned at $1064 \mathrm{~nm}, 8 \mathrm{~ns}$ pulse duration and an intensity around $40 \mathrm{MW} / \mathrm{cm}^{2}$. Mirrors are placed to guide the light and appropriate lenses are used to focus and image the beam. A half-wave plate, placed at the laser output, controls the polarization of the FF. A polarizer, positioned after the sample, controls the polarization of the SH. The sample is set in a rotary support in between two different filters. The first one eliminates background SH. This way we 
make sure that only FF (at $1064 \mathrm{~nm}$ ) enters the sample. The second one attenuates the FF that has passed through the sample. In addition, a HR mirror, a prism and a blocking edge are used in order to make sure that the signal we are measuring is all $\mathrm{SH}$ or TH. The efficiency of these PL signals is expected to be very low, of order $10^{-9}$ or $10^{-10}$. These values are far from what is usually measured and makes the detection very challenging. For this reason, light is collected with a photomultiplier so that the detected signal is strongly amplified. Then, it is analyzed with an oscilloscope. The transmitted PL harmonic signals are measured as a function of the angle of incidence by rotating the sample. This is not so simple in the reflection case as all the arm of detection has to be moved. In Fig. $2 \mathrm{~b}$ the encircled part in grey is fixed together, which makes us able to rotate it in order to measure reflection properly.

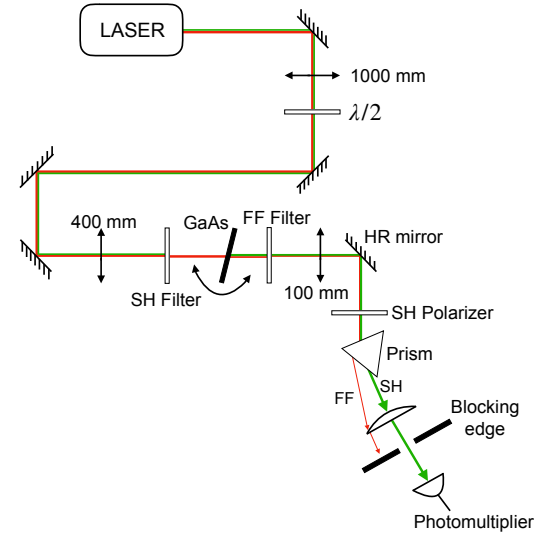

(a)

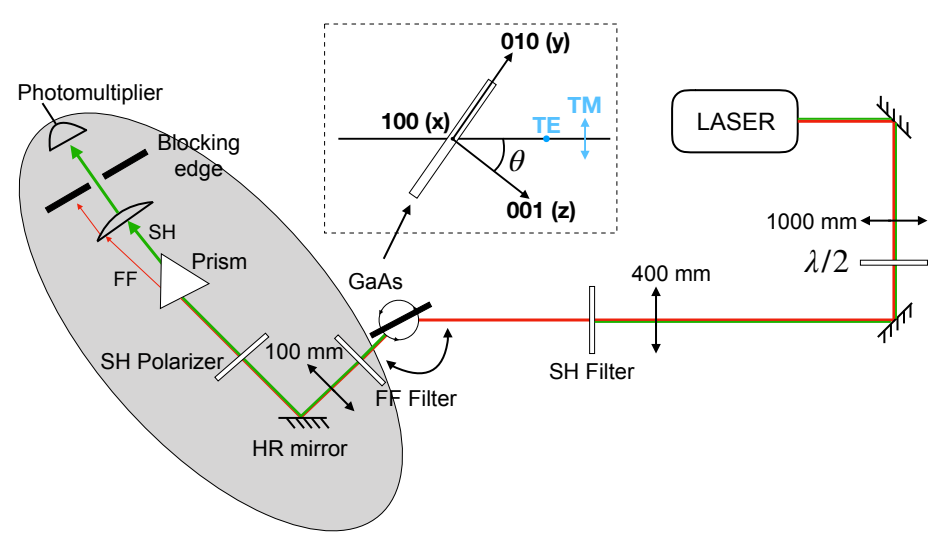

(b)

Figure 2: Transmission (a) and reflection (b) setups for measuring $\mathrm{SH}$ and $\mathrm{TH}$ signals.

So, there are three parameters that can be controlled. The polarization of the FF is changed by rotating the half-wave plate, the polarization of the $\mathrm{SH}$ is measured with the polarizer, and the angle of incidence of the $\mathrm{FF}$ to the sample is modified by rotating the support in which the substrate holds. We have focused the measurements on four different polarization combinations between the FF and the SH: TM-TM, TM-TE, TE$\mathrm{TM}$, TE-TE, as a function of the angle of incidence.

\section{Results and discussion}

\subsection{Numerical simulations}

In order to study the generation of $\mathrm{SH}$ in a GaAs substrate, several simulations have been performed. Our program allows us to approximately reproduce the experiment. It is based on the mathematical approach proposed in [5]. Briefly, this model solves Maxwell's equations taking into account the total nonlinear polarization shown in Eq. (6), so that bulk, Lorentz and surface contributions are taken into account in the generation of the harmonics. Through the simulation model we are able to modify input parameters such as angle of incidence, incident wavelength and polarization, pulse width and intensity, and the thickness of the GaAs substrate.

In Figs. 3a, 3b and 3c the simulated transmitted (blue) and reflected (red) PL SH 
efficiencies are plotted as functions of the angle of incidence. Incident wavelength and intensity are set to $1064 \mathrm{~nm}$ and $2 \mathrm{GW} / \mathrm{cm}^{2}$, respectively. Pulse-width is $50 \mathrm{fs}$ and the GaAs substrate is taken to be $10 \mu m$ thick. In Fig. 3a FF and SH fields are TM polarized. The transmission is maximum between $70^{\circ}$ and $80^{\circ}$, and it is approximately one order of magnitude larger than the reflection. A second peak in the reflected $\mathrm{SH}$ appears between $80^{\circ}$ and $90^{\circ}$. Fig. $3 \mathrm{~b}$ shows the $\mathrm{SH}$ efficiency when the $\mathrm{FF}$ and the $\mathrm{SH}$ field are TM and TE polarized, respectively. The TE-polarized SH signal is generated by the bulk $\chi^{(2)}$ of GaAs. Both transmission and reflection are two orders of magnitude smaller compared to the previous case illustrated in Fig. 3a and the maximum in transmission is now found around $50^{\circ}$. The TE-TM case illustrated in Fig. 3c refers to a TE-polarized pump and a surface-generated, TM-polarized SH signal. Transmission efficiency is of order $10^{-9}$ and the contrast in magnitude between transmission and reflection is not as large as in the previous cases. In both transmitted and reflected $\mathrm{SH}$ a peak appears at $80^{\circ}$. For TE-TE configuration, no $\mathrm{SH}$ is generated either in transmission and reflection. Fig. 3d shows the transmitted SH efficiency for each case.

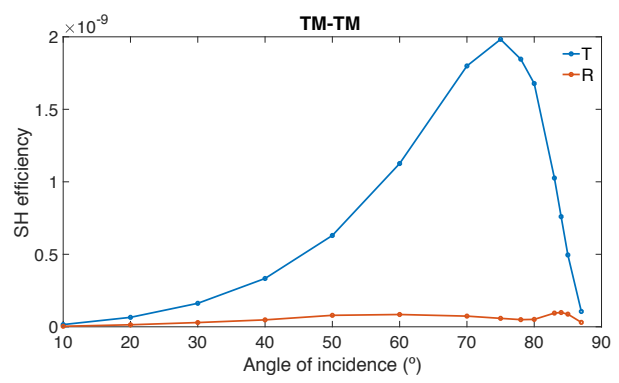

(a)

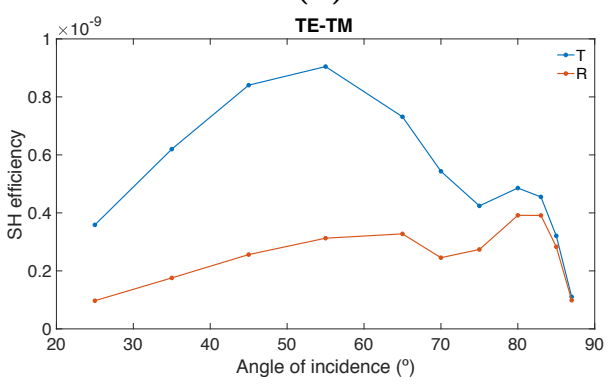

(c)

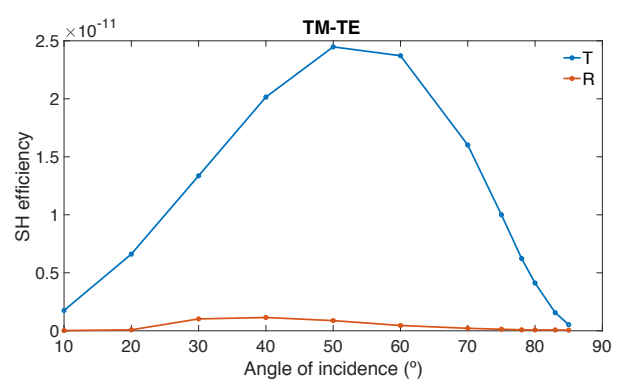

(b)

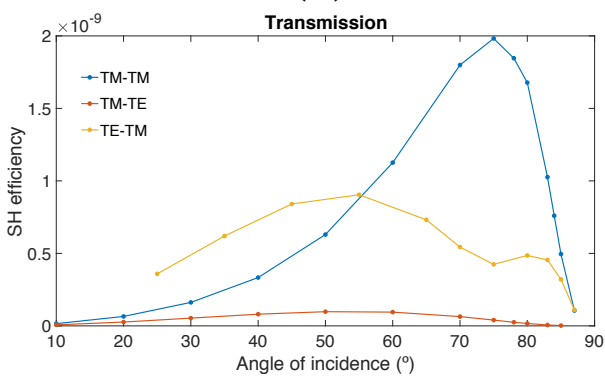

(d)

Figure 3: Calculated SH transmission (blue) and reflection (red) curves when bulk- and surfacegenerated $\mathrm{SH}$ are considered.

Figs. 3a, 3b, 3c and 3d were obtained by taking into account the three $\mathrm{SH}$ sources. An extensive study has been performed in order to see which source plays a role in the generation of each polarization of the SH. The analysis consisted in the activation and deactivation of each source term in each simulation. As a result, it was deduced that when the FF is TM-polarized, TM-SH arises from the surface and the Lorentz terms, while the TE-SH is generated by the bulk contribution. This is in agreement with Eq. (7). When the FF is TM-polarized $\left(\phi=0^{\circ}\right)$, the $\mathrm{SH}$ polarization coming from the bulk term is $\vec{P}(2 \omega)=d E_{0}^{2} \cos \theta \sin \theta \vec{i}$, so that TE-SH is obtained. Moreover, there is a maximum for $\theta=45^{\circ}$, which can also be seen in Fig. 3b. Notice also that for normal incidence $\left(\theta=0^{\circ}\right)$ the NL polarization becomes 0 so generation of the $\mathrm{SH}$ is not possible. 
When the FF is TE-polarized only TM-SH is generated and it is all due to the Lorentz term.

These phenomena were analyzed in [6], where a study of SHG and THG in centro-cymmetric semiconductors at visible and UV wavelengths in bulk and cavity environments was performed. In it, the complete NL second-order polarization vector for (001) GaAs is deduced. From it, it is demonstrated that when the incident light is TM-polarized, TM-SH is generated if the Lorentz and surface contributions are present. The same happens when the FF is TE-polarized and a SH field TM-polarized appears due to the presence of these surface terms. As far as the TH is concerned, a TMpolarized TH field appears when the incident light is TM-polarized due to the response of $\chi^{(3)}$. However, if the Lorentz and surface contributions are taken into account, a TE-polarized TH field is also generated.

\subsection{Experimental results}

In Figs. 4a, 4b and 4c the measured (blue) and simulated (red) transmitted SH efficiency as a function of $\theta$ is plotted for TM-TM, TM-TE and TE-TM polarizations, respectively. It can be seen that the measured and the simulated data fits quite well despite the oscillations that appear in the blue line which are going to be justified later. Fig. 4a and $4 \mathrm{c}$ demonstrate that not only the $\chi^{(2)}$ has to be taken into account but also the SHG at the surface. However, in our measurements we obtain efficiencies of the order of $10^{-9}$ and $10^{-10}$. For what the bulk contribution is concerned (TM-TE), the transmitted $\mathrm{SH}$ efficiency is proportional to $10^{-11}$, which tells us that the surface and Lorenz terms play a more important role in the generation of SH than the bulk for our GaAs sample. For TE-polarized FF and SH no signal was observed for any angle of incidence, which is also in accordance with the theoretical simulations. Fig. 4d shows the experimental data of transmitted SH efficiency for TM-TM, TM-TE and TE-TM configurations. It can be observed that the proportion between them is in agreement with Fig. 3d.

The $\chi^{(2)}$ (and $\chi^{(3)}$ ) parameter have been adjusted as well as the effective mass of the bound electrons so that the theoretical curve could fit the experimental data. The values of the NL susceptibilities are $10^{-11} \mathrm{~m} / \mathrm{V}$ and $10^{-20} \mathrm{~m}^{2} / \mathrm{V}^{2}$ respectively, while the effective mass is $0,0125 \mathrm{Me}$, where Me is the free electron mass. This is a nice result as, by analyzing the transmitted SH efficiency, properties of the GaAs substrate can be deduced.

Figs. 5a, 5b and 5c show the measured (blue) and simulated (red) reflected SH efficiency as a function of the angle of incidence. Fig. 5a fits almost perfect in shape, showing both the measured and the simulated curve a peak around $85^{\circ}$. In Fig. 5b it is observed that the experimental and the simulated data coincide in shape and order of magnitude. In Fig. 5c another peak appears in the measured reflection for large angles that are also present in the simulated curve. When the FF was set to be TE-polarized, no TE-SH was observed, which is also in accordance with the theoretical results. In Fig. 5d all the measured SH efficiency in reflection is collected.

Transmitted TH efficiency has also been measured as a function of $\theta$. Fig. 6a collects the measured (blue) and simulated (red) transmitted TH efficiency for TM-polarized FF. It can be seen that the oscillations are also present. For both curves the same behavior is observed, the larger the angle of incidence, the more decreases the TH efficiency. In Fig. $6 \mathrm{~b}$, the transmitted $\mathrm{TH}$ efficiency when the FF is TE-polarized is represented, showing 


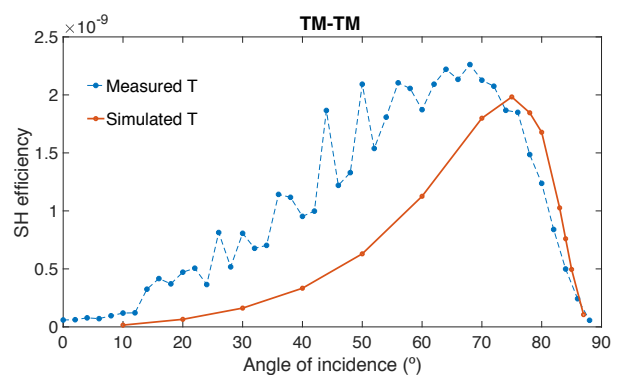

(a)

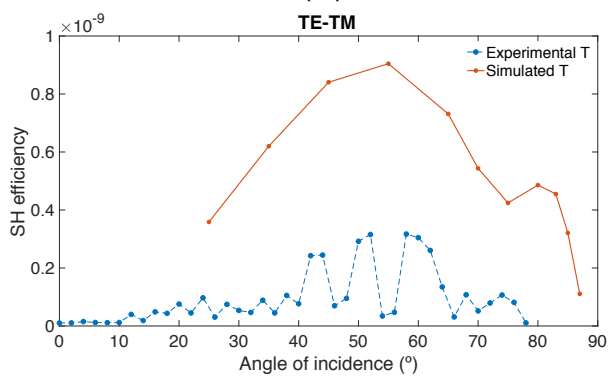

(c)

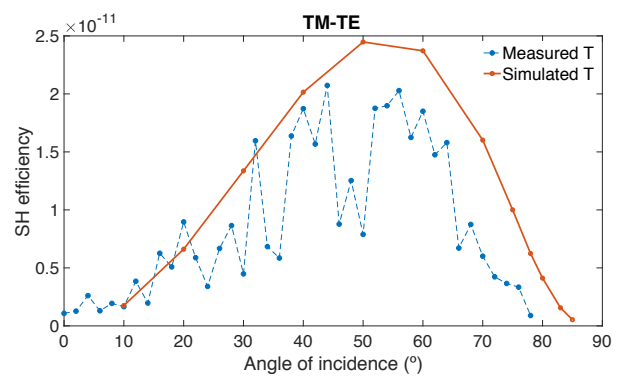

(b)

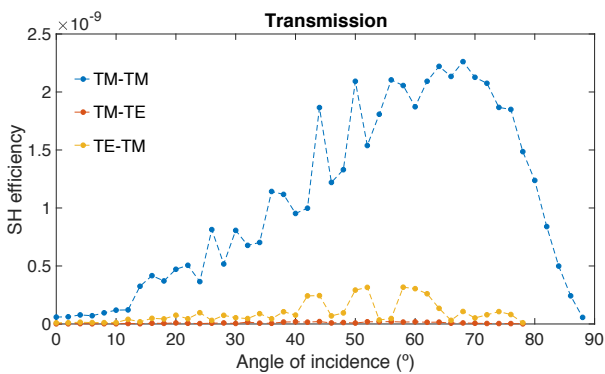

(d)

Figure 4: Calculated (red) and measured (blue) SH transmission efficiencies as a function of the angle of incidence.

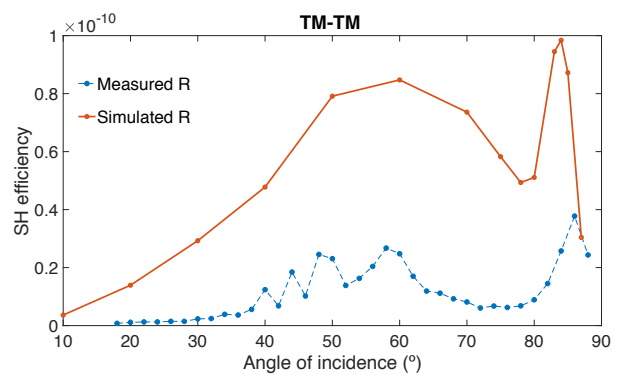

(a)

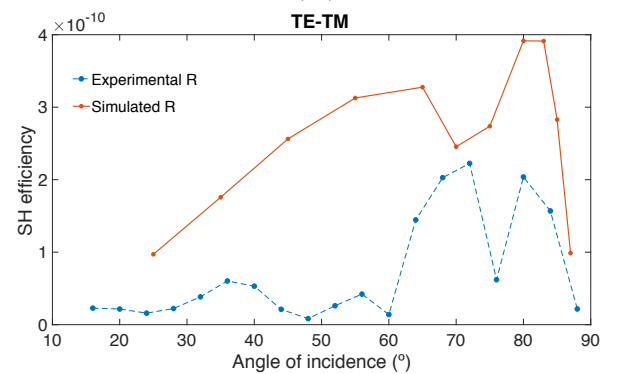

(c)

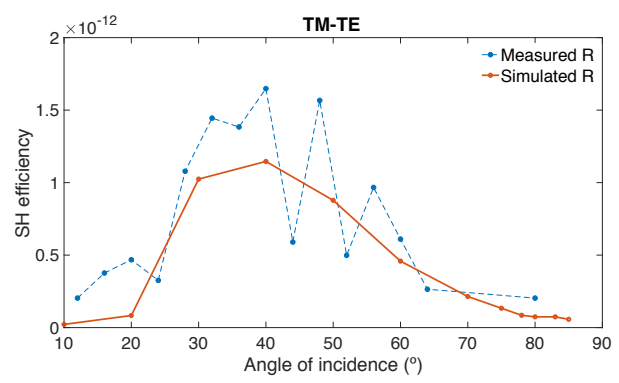

(b)

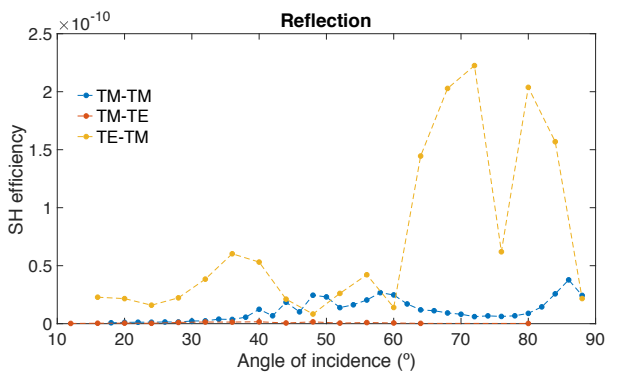

(d)

Figure 5: Calculated (red) and measured (blue) SH reflection efficiencies as a function of the angle of incidence.

the same behavior as in the previous case. It is important to notice here that we have been able to measure efficiencies of the order of $10^{-13}$ thanks to the good functioning of our setup (Fig. 2a).

In all the measured data we have seen oscillations in both transmission and reflection. 
In order to analyze them we performed more accurate measurements (one of which can be observed in Fig. 6a), and approximately 1, 4 degrees/oscillation were counted. This resonances are explained due to the fact that for different angles of incidence, the path length that the light has to travel from the entrance to the exit of the sample changes. In going from normal incidence to $\theta=90^{\circ}, 1,25$ degrees/oscillation are calculated, which is in agreement with what we have got in our measurements. Moreover, more or less the same number of oscillations are obtained for the $\mathrm{SH}$ and the $\mathrm{TH}$ meaning that these are resonances of the FF. So, the GaAs substrate is acting as a resonant cavity and that is why these oscillations appear. If a thinner substrate was used, the path length would not change as much with the angle of incidence and these resonances would banish.

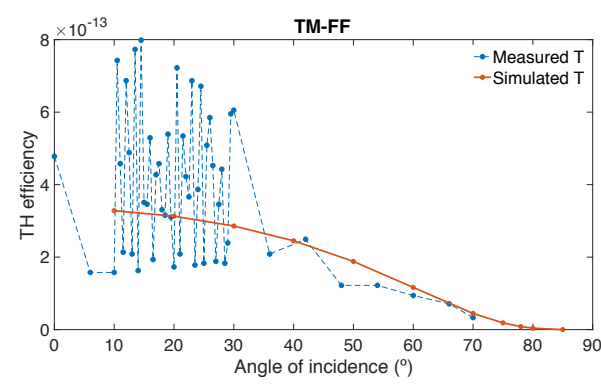

(a)

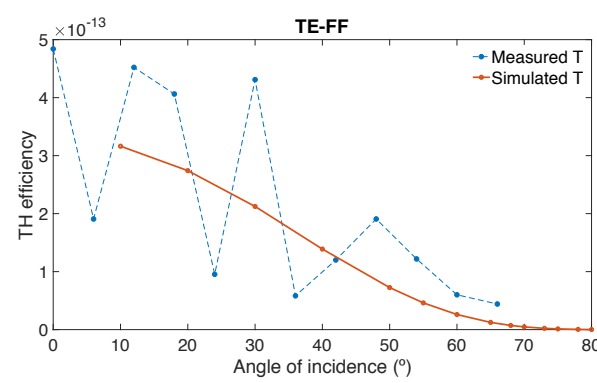

(b)

Figure 6: Calculated (red) and measured (blue) TH transmission efficiencies as a function of the angle of incidence.

In order to conclude the results we took measurements of the TM and TE-polarized $\mathrm{SH}$ efficiency as a function of the FF polarization when $\theta$ was fixed at $45^{\circ}$. This is what is represented in Figs. 7a and 7b for TM and TE-polarized SH, respectively. The experimental data (blue) was fitted to a curve (red) of the form:

$$
\frac{E(2 \omega)}{E(\omega)}=\left(\alpha \sin (2 \phi)+\beta \cos ^{2} \phi+\gamma\right)^{2}
$$

which represents the interference between the bulk, the surface and the Lorentz terms, and $\alpha, \beta$ and $\gamma$ are constants. For instance, for TM-polarized SH we have seen that the bulk contribution is proportional to $\sin (2 \phi)$ and the surface term is proportional to $\cos ^{2} \phi$. For what the Lorentz term is concerned, we have taken it as a constant since the $\vec{E} \times \vec{H}$ polarization is longitudinal, so the SH due to the Lorentz force will always lie in the plane of incidence regardless of the angle $\phi$. The fittings for the TM (Eq. (9)) and TE (Eq. (10)) polarized SH are the following ones:

$$
\begin{aligned}
& \frac{E(2 \omega)}{E(\omega)}=1,1064 \cdot 10^{-9}\left(-0,08938 \sin (2 \phi)+1,36 \cos ^{2} \phi-0,3756\right)^{2} \\
& \frac{E(2 \omega)}{E(\omega)}=1,3636 \cdot 10^{-10}\left(0,9512 \sin (2 \phi)+0,2992 \cos ^{2} \phi-0,1012\right)^{2}
\end{aligned}
$$

As it can be seen, the surface contribution in both cases $\left(\cos ^{2} \phi\right.$ for TM-SH and $\sin (2 \phi)$ for TE-SH) is larger than the bulk $\left(\sin (2 \phi)\right.$ for TM-SH and $\cos ^{2} \phi$ for TE-SH). So with this, we corroborate that the surface term is the main source of generation of $\mathrm{SH}$ in our GaAs substrate. 


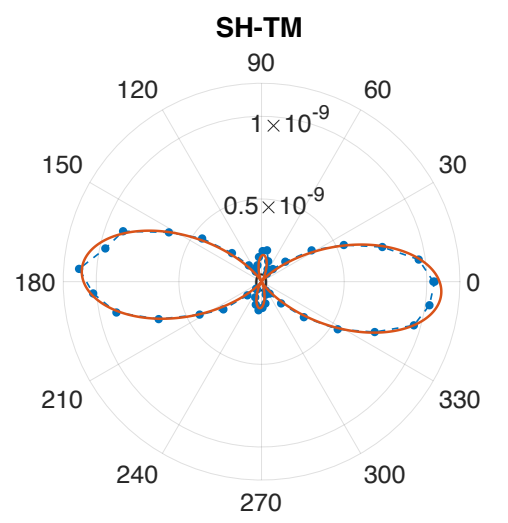

(a)

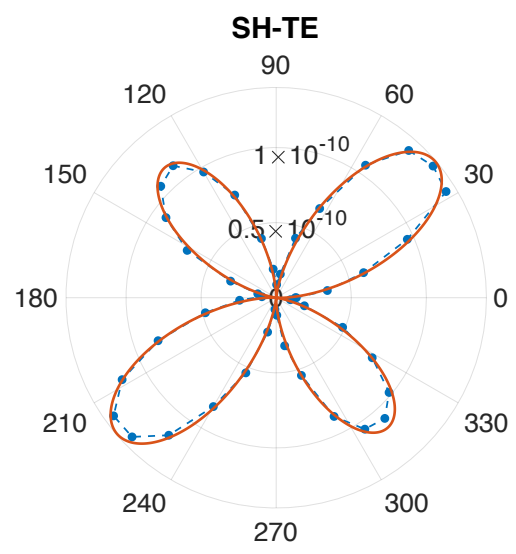

(b)

Figure 7: Transmission measurements (blue) and fitting (red) of the SH efficiency when the electric field is rotated.

\section{Conclusions}

We have proved with these preliminary results SHG and THG in the opaque region of GaAs, which demonstrates that the SH and TH pumps are PL. Moreover, it has been shown that the experimental data fits well theoretical predictions despite resonances appearing in the measured curves which have been justified. This fact gives us information about the sample as the fitting has been done by adjusting the secondand third-order NL susceptibilities and the effective mass of bound electrons.

We were able to measure transmission efficiencies of order $10^{-9}-10^{-11}$ for the $\mathrm{SH}$ and of order $10^{-13}$ for the TH. Although propagation phenomena and NL frequency conversion below the absorption edge of semiconductors is still lacking primarily because these processes are thought to be uninteresting and inefficient due to absorption and to the naturally high degree of phase mismatch, harmonic generation in absorbing materials can be useful for realizing coherent sources and is interesting because of the many potential applications that semiconductors find in optical technology.

\section{References}

[1] J. A. Armstrong, N. Bloembergen, J. Ducuing, and P. S. Pershan, Interactions between Light Waves in a Nonlinear Dielectric Phys. Rev. 127, 1918 (1962)

[2] L. D. Noordam, H. J. Bakker, M. P. de Boer, and H. B. van Linden van den Heuvell, Secondharmonic generation of femtosecond pulses: observation of phase-mismatch effects Opt. Lett. 15, $24(1990)$

[3] Marco Centini, Vito Roppo, Eugenio Fazio, Federico Pettazzi, Concita Sibilia, Joseph W. Haus, John V. Foreman, Neset Akozbek, Mark J. Bloemer, and Michael Scalora, Inhibition of Linear Absorption in Opaque Materials Using Phase-Locked Harmonic Generation Phys. Rev. Lett. 101, 113905 (2008)

[4] E. D. Palik, Handbook of Optical Constants of Solids Academic, New York (1985)

[5] M. Scalora, M. A. Vincenti, D. de Ceglia, V. Roppo, M. Centini, N. Akozbek, and M. J. Bloemer Second- and third-harmonic generation in metal-based structures Phys. Rev. A 82, 043828 (2010)

[6] M. Scalora, M. Vincenti, D. de Ceglia, N. Akozbek, V. Roppo, M. Bloemer and J. W. Haus, Dynamical model of harmonic generation in centrosymmetric semiconductors at visible and UV wavelengths, Phys. Rev. A 85, 053809 (2012). 